\title{
UV causes dramatic changes in aggregation with mixtures of photo-active and inert surfactants
}

\author{
Supporting material
}

\section{Julian Eastoe*, Margarita Sanchez Dominguez, Paul Wyatt and Andrew Orr-Ewing School of Chemistry, University of Bristol, Bristol, BS8 1TS UK.}

Tel. : UK + 1179289180 fax : UK + 1179250612 e-mail : julian.eastoe@bristol.ac.uk

\section{Richard K. Heenan}

ISIS-CLRC, Rutherford Appleton Laboratory, Chilton, OXON, OX11 0QX UK

\section{SANS theory}

References 1 to 8 cover the relevant theory and scattering laws [1-8], reference 1 gives details on the FISH fitting program (available from rkh@isis.rl.ac.uk). The SANS scattering cross section for a reasonably dilute system of $N_{P}$ particles is given by the product of a form, or shape, factor $P(Q)$ and an inter-particle structure factor $S(Q)$.

$\frac{d \Sigma(Q)}{d \Omega}=N_{p} P(Q) S(Q)$

For a dilute, non-interacting system $S(Q)$ tends to unity and may be ignored, else wise it may be calculated for hard spheres in the Percus-Yevick approximation or for charged spheres in the RMSA approximation [2]. Here $S(Q)$ was also corrected for the effects of polydispersity using the method of Chen et al. [3]. For a polydisperse system of particles the form factor is simply summed over a size distribution $\mathrm{N}(\mathrm{R})$.

$P(Q)=\left(\rho_{p}-\rho_{m}\right)^{2} \int N(R) F^{2}(Q, R) d R$

For a sphere of radius $R, F(Q, R)=f(Q, R)$ and is given by

$$
f(Q, R)=V(R) \frac{3(\sin (Q R)-Q R \cos (Q R))}{(Q R)^{3}}
$$

where $V(R)=\frac{4 \pi}{3} R^{3}$. For a hollow spherical shell of inner radius $r$ and thickness $\Delta$

$$
F(Q, r)=f(Q, r)-f(Q, r+\Delta)
$$

In either case the total volume of the dispersed particle may be simply computed given absolute scattered intensities and the neutron scattering length densities of the particle $\rho_{p}$ and solvent medium $\rho_{\mathrm{m}}$. In order to calculate $\rho_{\mathrm{p}}$ and surfactant volume fraction, the appropriate molecular volumes of the different surfactants were used. For DTAB, an average value of $480 \AA^{3}$ was obtained from combining those of Bergström and Pedersen [9], $491 \AA^{3}$, and Wettig and Verral [10], 479.1 $\AA^{3}$. For 12-4-12, Wettig and Verral [10] have reported a value of $971.7 \AA^{3}$, which was obtained by using the additivity method from Gianni et al [11]. For 16-4-16, the molecular 
volume was calculated by adding the contribution of eight methylene groups $\left(\mathrm{V}_{\mathrm{m}}\right.$ of $\mathrm{CH}_{2}=26.5$ $\AA^{3}$ ) to the molecular volume of $12-4-12$, to obtain a value of $1183.7 \AA^{3}$. For E-SGP, a value of $1578 \AA^{3}$ was obtained by adding the partial molar volumes of the different functional groups present in the molecule [12], and converting that into molecular volume.

For a large radius, rigid, hollow sphere or vesicle the form factor $P(Q)$ has a characteristic oscillation caused by interference between scattering from opposite faces of the sphere. A "free form" distribution $\mathrm{N}(\mathrm{R})$ for vesicle radii was used to cover the range $r=50$ to about $500 \AA$ with ten adjustable height "sticks", the distribution $N(R)$ being made smooth and continuous by a local cubic interpolation between an adjacent group of four "sticks" (any negative values being set zero). The value of $N(R)$ at the first and last sticks was also kept zero, leaving essentially eight adjustable parameters. An essentially bimodal distribution of vesicle radii provided a significantly better fit to the oscillations at small $Q$ for $X_{S G P} \geq 0.75$ whilst only one population was observed for $X_{S G P}=0.25-0.5$. Vesicle radii distribution for all samples are shown in Figure 1 below.

Prior to irradiation the $\mathrm{Q}^{-2}$ scattering was consistent with vesicles (sheets) [e.g. 1,8] in equilibrium with much smaller charged micelles. SANS from the post-irradiated samples could be interpreted either as monodisperse prolate ellipsoidal charged micelles or as Schultz spherical charged micelles with a very low polydispersity. Structure factor of irradiated samples was fitted as described by the Hayter-Penfold approach [2, 4-6] for both models.

Owing to the large number of (possible) free parameters in the vesicle-spheres combined model, some of these were input as constants, based in initial trial fits: vesicle layer thickness $20 \AA$, (24 $\AA$ for $16-4-16, X_{S G P}=0.5$ and 0.75$)$, micelle radius $20 \AA$, (allowed to fit for $12-4-14 X_{S G P}$ $=0.25$ and $16-4-16 X_{S G P}=0.5$ and 0.75$)$. The distribution in vesicle radius $N(R)$, was allowed to vary, as was the fraction of aggregates present as vesicles (Table 3 in the main manuscript).

For the prolate ellipsoidal charged model, the Debye length was input as a constant based on the composition of the systems [1]: $18 \AA$ for DTAB mixtures, and $25 \AA$ for $12-4-12$ and 16-4-16 mixtures. Surfactant volume fraction was also input as constant based on composition of the known/estimated molecular volumes. Semiminor axis a, charge and Hayter-Penfold radius were allowed to vary, and several values for the axial ratio $\mathrm{x}$ were tested until a satisfactory scale factor was obtained. A similar approach was followed for spheres, except that the fitting parameters were sphere radius, charge, Hayter-Penfold radius and polydispersity. Absolute intensity scale factor agreement was always better for ellipsoidal micelles fits. The fractional molecular charge $\alpha$ was calculated from charge and Hayter-Penfold radius [1, 2, 4-6], the known composition and molecular volumes in the mixtures. Table 1 below shows values of $\alpha$ for ellipsoidal and spherical micelles fits. 
Figure 1. Fitted vesicle size distributions for SGP and (a) DTAB (b) 12-4-12 and (c) 16-4-16 mixtures: $(\bullet \bullet \bullet) X_{S G P}=0.25,(-\cdots) X_{S G P}=0.5,(--) X_{S G P}=0.75,(-) X_{S G P}=1$.

Figure 1 - Eastoe et al
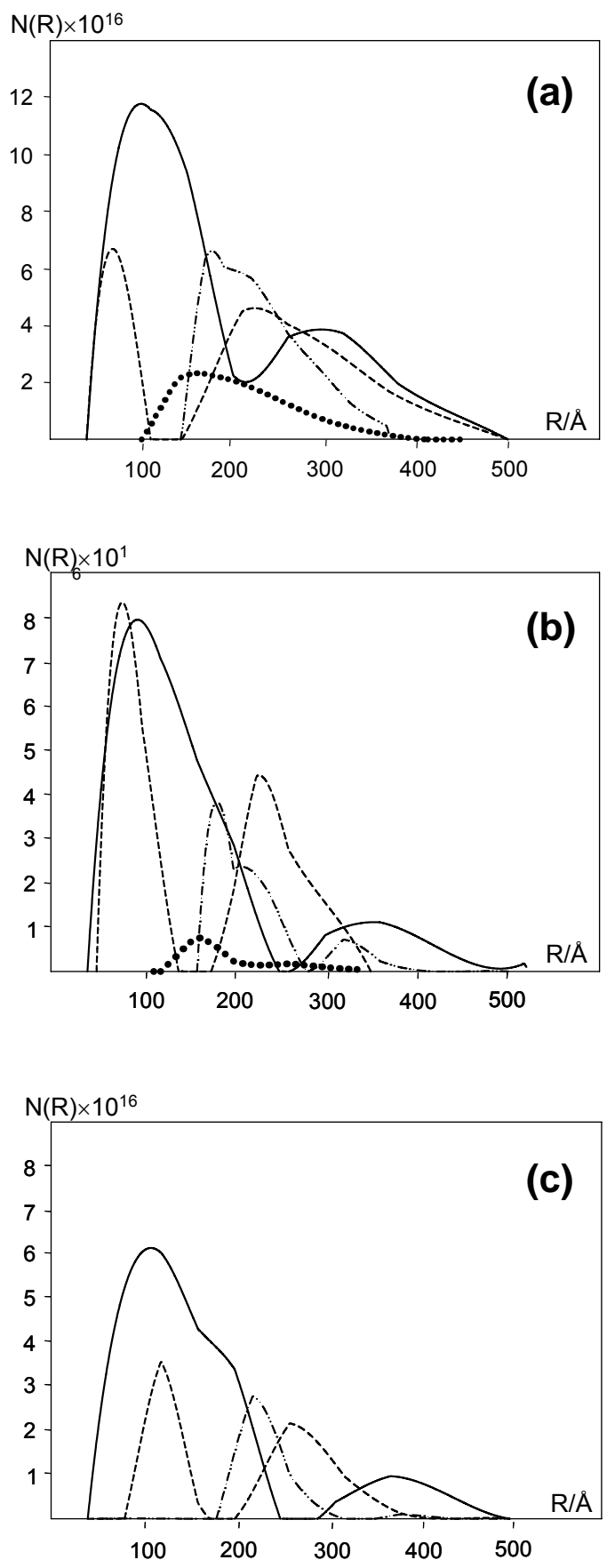
Table 1. $S(Q)$ of irradiated samples: fractional molecular charge $\alpha$ as obtained by fitting SANS data to models of either prolate ellipsoidal or spherical micelles.

\begin{tabular}{|c|c|c|c|}
\hline MIXER & $\mathrm{X}_{\mathrm{SGP}}$ & $\begin{array}{c}\alpha \\
\text { ellipsoidal } \\
\text { micelles }\end{array}$ & $\begin{array}{c}\alpha \\
\text { spherical } \\
\text { micelles }\end{array}$ \\
\hline \multirow[t]{5}{*}{ DTAB } & 0.0 & 0.22 & 0.26 \\
\hline & 0.25 & 0.31 & 0.32 \\
\hline & 0.50 & 0.37 & 0.38 \\
\hline & 0.75 & 0.19 & 0.45 \\
\hline & 1.0 & 0.18 & 0.48 \\
\hline \multirow[t]{5}{*}{$12-4-12$} & 0.0 & 0.20 & 0.25 \\
\hline & 0.25 & 0.26 & 0.28 \\
\hline & 0.50 & 0.29 & 0.31 \\
\hline & 0.75 & 0.31 & 0.34 \\
\hline & 1.0 & 0.36 & 0.39 \\
\hline \multirow[t]{5}{*}{$16-4-16$} & 0.0 & 0.42 & - \\
\hline & 0.25 & 0.31 & 0.37 \\
\hline & 0.50 & 0.34 & 0.35 \\
\hline & 0.75 & 0.37 & 0.40 \\
\hline & 1.0 & 0.39 & 0.43 \\
\hline
\end{tabular}




\section{References}

1.Heenan, R. K. FISH Data Analysis Program; Rutherford Appleton Laboratory; Report RAL89-129; CCLRC: Didcot, U.K., 1989.

2.Hayter, J.B.; Penfold, J. J. Chem. Soc., Faraday Trans 1 1981 77, 1851.

3.Kotlarchyk, M.; Chen, S.-H.; Huang, J. S.; Kim, M. W. Phys. Rev. A. 1984, 29, 2054

4. Hayter, B.J. Mol. Phys. 1981 42, 109.

5. Hayter, B.J. Mol. Phys. 1982 46, 651.

6. Hayter, J. B.; Penfold, J. Colloid Polym. Sci. 1983 261, 1022.

7.Livesey, I. J. Chem. Soc., Faraday Trans 21987 83, 1445.

8.Eastoe, J. New Physico-Chemical Techniques for the Characterisation of Complex Food Systems; Dickenson, E., Ed.; Blackie: Glasgow 1995; p 268.

9.Bergström, M.; Pedersen, J. S. Phys. Chem. Chem. Phys. 1999 1, 4437.

10. Wettig, S. D.; Verral, R. E. J. Colloid and Interface Science 2001 235, 310.

11. Gianni, P.; Lepori, L. J. Solution Chemistry 1996 25, 1.

12. Gianni, P.; Lepori, L. J. Solution Chemistry 2000 29, 405. 